\title{
CONSTRUCTION AND TESTING OF ARC DIPOLES AND QUADRUPOLES FOR THE RELATIVISTIC HEAVY ION COLLIDER (RHIC) AT BNL*
}

\author{
P. Wanderer, J. Muratore, M. Anerella, G. Ganetis, A. Ghosh, A. Greene, R. Gupta, A. Jain, S. Kahn, \\ E. Kelly, G. Morgan, A. Prodell, M. Rehak, W. Sampson, R. Thomas, P. Thompson, E. Willen \\ Brookhaven National Laboratory, Upton, New York 11973-5000 USA
}

\begin{abstract}
The production run of superconducting magnets for the Relativistic Heavy Ion Collider (RHIC) project at Brookhaven National Laboratory (BNL) is well underway. Or the 288 arc dipoles needed for the collider, more than 120 have been delivered. More than 150 arc quadrupoles have been delivered. All of these magnets have been accepted for RHIC. This paper reports the construction and performance of these magnets. Novel features of design and test, introduced to enhance technical performance and control costs, are also discussed. Other papers submitted to this Conference summarize work on the sextupoles and tuning quads [1], arc correctors [2], and combined correctorquadrupole-sextupole assemblies (CQS) [3].
\end{abstract}

\section{ARC DIPOLES}

\section{A. Design and Construction}

The arc dipole cold mass, shown in Figure 1, has a measured central field of $3.40 \mathrm{~T}$ at $5.0 \mathrm{kA}$ operating current, an effective length of $9.44 \mathrm{~m}$, and an $80 \mathrm{~mm}$ bore. The $\mathrm{NbTi}$ filaments have a diameter of $6 \mu \mathrm{m}$, with a minimum critical current density of $2600 \mathrm{~A} / \mathrm{mm}^{2}$ at $5 \mathrm{~T}, 4.2 \mathrm{~K}$. The cable is made up of 30 wires with diameter $0.648 \mathrm{~mm}$ [4]. The 32-turn single-layer coil uses three wedges to achieve good field quality.

The magnet includes several novel features designed to reduce cost and improve quality. The turn-to-turn insulation of the cable is a double wrap of Kapton CI [5] polyimide with a polyimide adhesive on the outside of the outer wrap. This insulation offers numerous advantages, including better protection against punch-through and coils with better azimuthal size uniformity than previous wraps using fiberglass [6]. When the coils are molded, the high temperature necessary to set the adhesive is applied only briefly ( $225 \mathrm{C}$ for $5 \mathrm{~min}$. at minimum pressure) to avoid degradation of interstrand resistance. The high pressure used to size the coils is applied as a separate step at lower temperature ( $140 \mathrm{C}$ for $30 \mathrm{~min}$.). The azimuthal sizes of the coils selected for a magnet differ by at most $25 \mu \mathrm{m}$.

Another novel feature is the use, in three places, of injection-molded parts. An RX630 [7] phenolic spacer located between the coil and the yoke defines the pole angle, provides coil insulation to ground, and reduces saturation effects. The coil end saddles and spacers are made of high-temperature Ultem 6200 [8] able to tolerate deformation due to the high temperatures of the coil cure cycle. The three posts that support the cold mass in the cryostat are made of Uiltem 2100 .

\footnotetext{
Work supported by the U.S. Department of Energy under Contract No. DE-AC02-76CH00016.

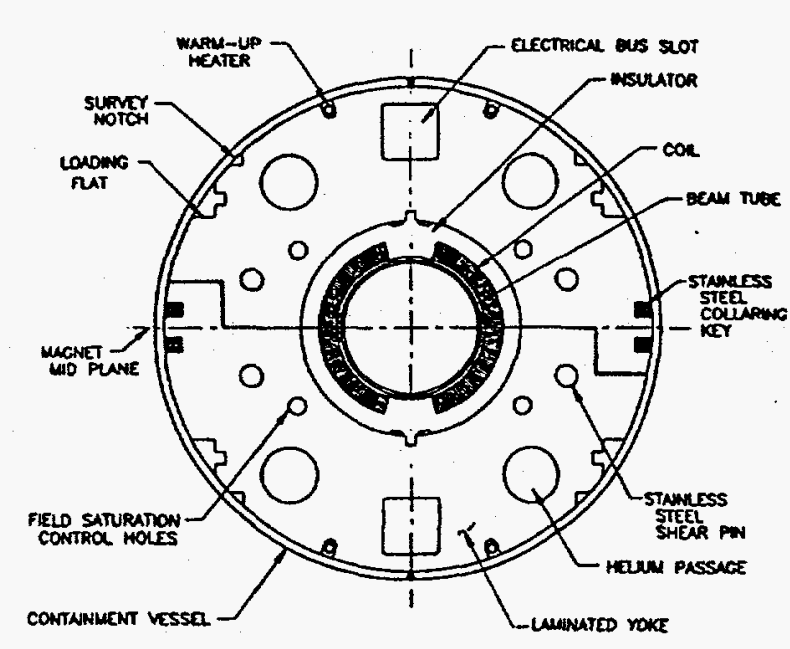

Fig. 1. Cross section of RHIC arc dipole cold mass.

Also novel is the use of the iron yoke as a collar for the coil, providing both prestress and flux return. The yoke laminations are $6.35 \mathrm{~mm}$ thick (to allow their use as collars), with dimensional precision of $25 \mu \mathrm{m}$ maintained by the use of fine blanking. The cold mass sagitta is large, $46 \mathrm{~mm}$. A more detailed description of the magnet is given elsewhere [9].

The dipoles are being manufactured by the Northrop Grumman Corporation (NGC) ready for tunnel installation, under a build-to-print contract. A total of $37380 \mathrm{~mm}$ dipoles have been ordered: $2989.44 \mathrm{~m}$ dipoles (including 10 spares) and 75 shorter dipoles with the same cross section for use in the insertion regions. The delivery rate is now one per day, with the entire order scheduled for completion in June 1996. A more detailed account of the manufacturing process is in preparation [10].

\section{B. Test Procedures and Results}

Tests performed on all dipoles at room temperature include hipot, optical survey, and field quality. The harmonics and field angle are measured at the vendor's site with a system containing a $1 \mathrm{~m}$-long rotating coil and gravity sensors ("mole") supplied by BNL [11]. The integral field is measured with a stationary coil, by ramping the magnet. Field quality measurements are made at currents up to $30 \mathrm{~A}$.

The horizontal and vertical field components, $B_{x}$ and $B_{y}$ throughout the magnet aperture are given in polar coordinates $(r$, Q) by 


$$
B_{y}+i B_{x}=10^{-4} B(R) \sum_{n=0}^{\infty}\left(b_{n}^{\prime}+i a_{n}^{\prime}\right)(\cos n \theta+i \sin n \theta)\left[\frac{r}{R}\right]^{n}
$$

where $I$ is the imaginary unit and $B(R)$ is the magnitude of the field due to the fundamental at the reference radius $(R)$. In a normal dipole, $b_{0}=10^{4}$ and $B(R)=B_{0}$ In a normal quadrupole, $b_{1}=10^{4}$ and $B(R)=G R$ where $G$ is the gradient $\partial B_{y} / \partial x$ at the magnet center.

Each of the initial 33 dipoles was quench-tested at BNL. Quench location data from an antenna system were taken for nearly all magnets. Field integrals were measured near injection $(660 \mathrm{~A})$, transition (1450 A), and store (5 kA). A number of magnets had additional tests to check the quench performance after thermal cycling and the AC and time-dependent properties of the superconductor. Fortunately, for RHIC operation, cable timedependent characteristics are not significant.

Following careful review of the cold-test results from these dipoles and of the correlation between the field quality measured at room temperature ("warm") and cold, the RHIC Project implemented a plan to reduce the cost of cryogenic testing by cold-testing only $10 \%$ of the remaining magnets. Every tenth dipole is nominally scheduled for cold test. However, magnets with unusual construction histories are added to or substituted for the nominal magnets as the occasion arises.

Dipoles were cold-tested at $4.6 \mathrm{~K}, 5 \mathrm{~atm} \mathrm{He}$ with a mass flow rate of $100 \mathrm{~g} / \mathrm{sec}$. Conductor-limited quenches were calculated from cable short-sample data to lie in the range $6.9 \mathrm{kA}$ $7.3 \mathrm{kA}$. The initial ramp to quench was made at $25 \mathrm{~A} / \mathrm{sec}$, with pauses of 0.5 minute each $500 \mathrm{~A}$ (to monitor bus and lead stability), except for a 1 hour wait at $5.5 \mathrm{kA}$ (to check that the magnets would operate at the RHIC power supply limit without quenching). No magnet has quenched below $5 \mathrm{kA}$. Subsequent ramps to quench were at the design ramp rate of $83 \mathrm{~A} / \mathrm{sec}$. Typically magnets were quenched six times, with the last four quench currents lying in a narrow range ("on plateau"), at the expected conductor limit.

Dipoles quenching within a 100 A range above $6.5 \mathrm{kA}$ (30\% margin) are accepted automatically. Dipoles quenching in the range $6.3 \mathrm{kA}-6.5 \mathrm{kA}$ are reviewed individually. All 41 coldtested dipoles have been accepted; two have been reviewed individually. A summary of the plateau quench currents of all the magnets is given in Figure 2.

The integral transfer function, measured with the stationary coil at room temperature, is plotted against the dipole production sequence number in Figure 3. The accelerator requirement is that the rms fractional variation in the transfer function be less than $0.05 \%$. For the 119 magnets in the figure, the fractional variation is $0.03 \%$. Several features of the history catch the eye. First, the decrease at number 10 , which occurred when NGC switched from BNL-supplied RX630 spacers to their own supplier. Second, the decrease at number 89 , which investigation determined to be associated with the RX630 spacers, some of which had insufficient radial thickness. Beginning with number 108 , each RX630 piece was inspected to insure sufficient radial thickness. RX630 pieces now being supplied have sufficient radial thickness. Magnets with low transfer function have been paired with magnets with high transfer function to reduce the load

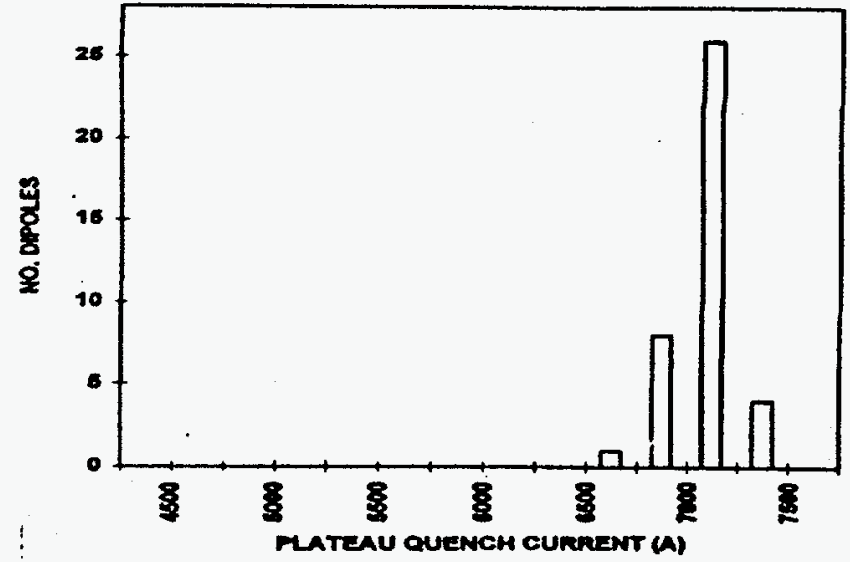

Fig. 2. Distribution of plateau quench currents of RHIC arc dipoles

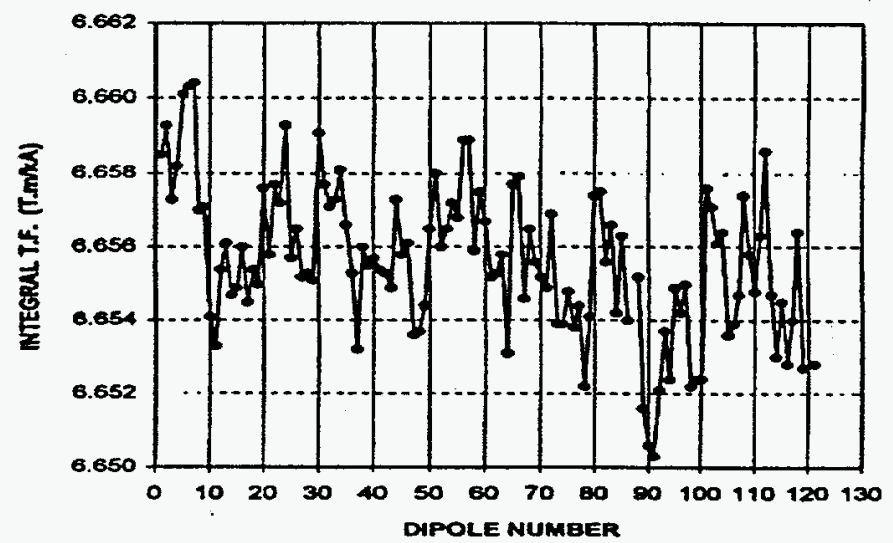

Fig. 3. Trend plot of arc dipole integral transfer function measured warm

on the trim dipoles, as described in another paper to this Conference [12]

The correlation between the warm and cold measurements of the integral transfer function is shown in Figure 4. The average ratio of the warm to the cold measurements of the transfer function is about 0.96 . The magnet indicated by the solid symbol was excluded from the average. The ms variation of this ratio, $0.02 \%$, characterizes the uncertainty in calculating the cold transfer function from warm measurements and is sufficiently small that the accelerator requirement, $0.05 \%$, can be assured. The success of the stationary coil for warm, low-field measurements was essential, since NMR cannot be used at such low fields.

Integral values of the dipole angle have all been less than $3.2 \mathrm{mrad}$, averaging $-0.4 \mathrm{mrad}$. Up to $5 \mathrm{mrad}$ offset can be compensated during magnet installation into RHIC. The correlation between warm and cold measurements of the dipole angle is shown in Figure 5. Magnets with identical warm and cold measurements would lie on the line. The rms variation of the difference between warm and cold measurements is $0.15 \mathrm{mrad}$, much smaller than the accelerator tolerance of $1 \mathrm{mrad}$ for installed magnets. 


\section{DISCLAIMER}

Portions of this document may be illegible in electronic image products. Images are produced from the best available original document. 


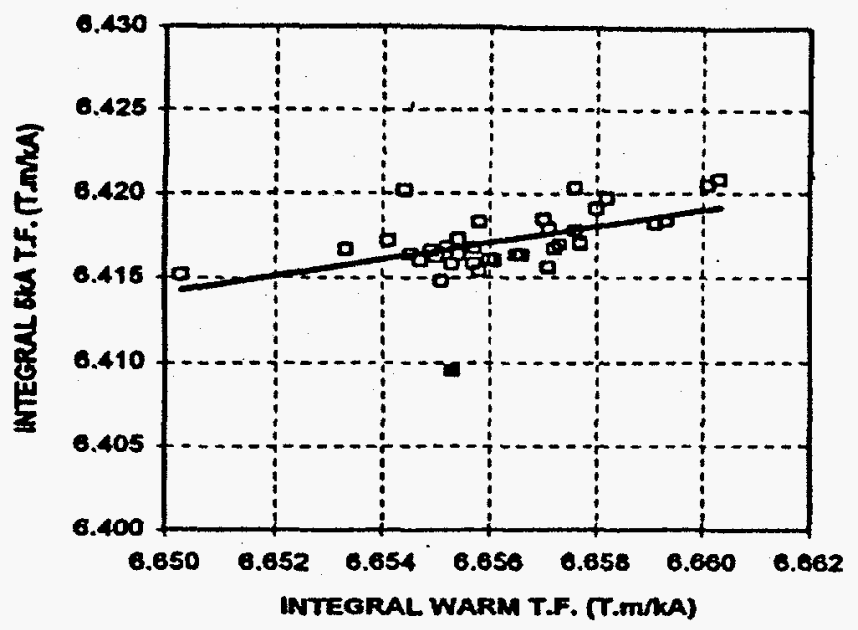

Fig. 4. Correlation of warm and $5 \mathrm{kA}$ measurements of arc dipole integral transfer function

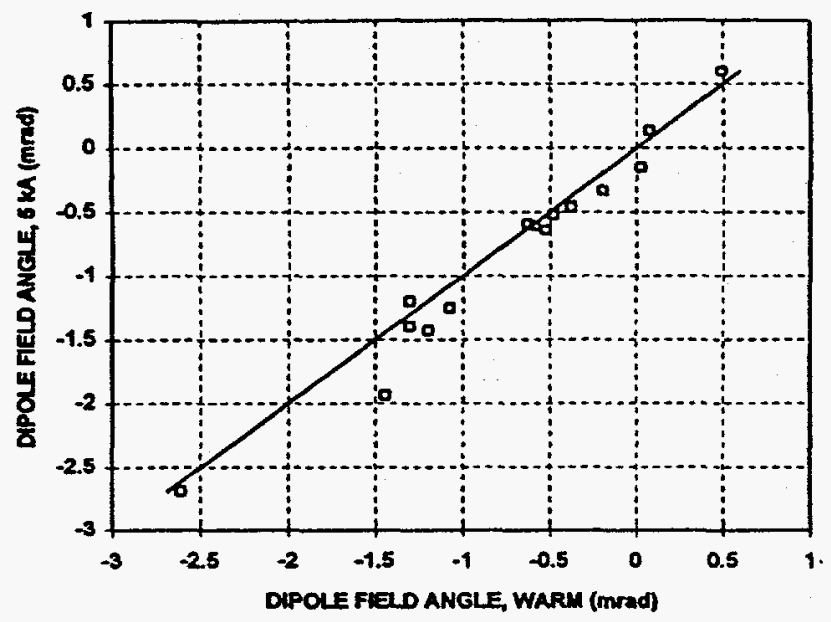

Fig. 5. Correlation of warm and $5 \mathrm{kA}$ measurements of arc dipole integral field angles

The dipole twist is characterized by the rms variation of the eight measurements made with the mole in the straight section of the magnet during an axial scan. An increase can be noted for magnet numbers 83 through 90 (Figure 6). It appears to have been associated with a temporary change in the method used to obtain the proper sagitta. Discussions of the twist with NGC at this time resulted in an overall decrease. The allowed value of twist is $3 \mathrm{mrad}$.

The skew quadrupole term $a_{1}$ is an important indicator of the quality of magnet construction since it is produced by top bottom asymmetries in the magnet. The most common of these is the difference in the azimuthal sizes of the upper and lower coils, for which the sensitivity is about 2 units of $a_{1}$ per $25 \mu \mathrm{m}$ of difference. The skew quadrupole is plotted as a function of magnet sequence number in Figure 7. The mean $a_{1}$ is -0.02 units, with an rms variation of 1.4 units. These compare favorably to the values estimated for the series production, a mean of 1 unit or less, rms of 1.3 units. (The large value of $a_{1}$ in dipole number 105 is due to the use of coils which differed in size by about $75 \mu \mathrm{m}$. The pairing of these coils was forced by

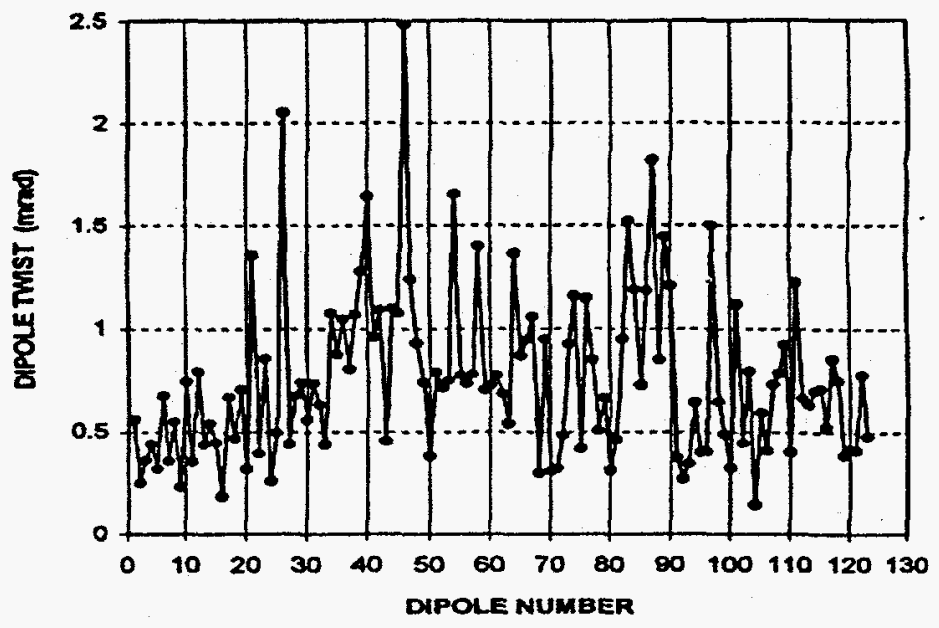

Fig. 6. Trend plot of the arc dipole twist

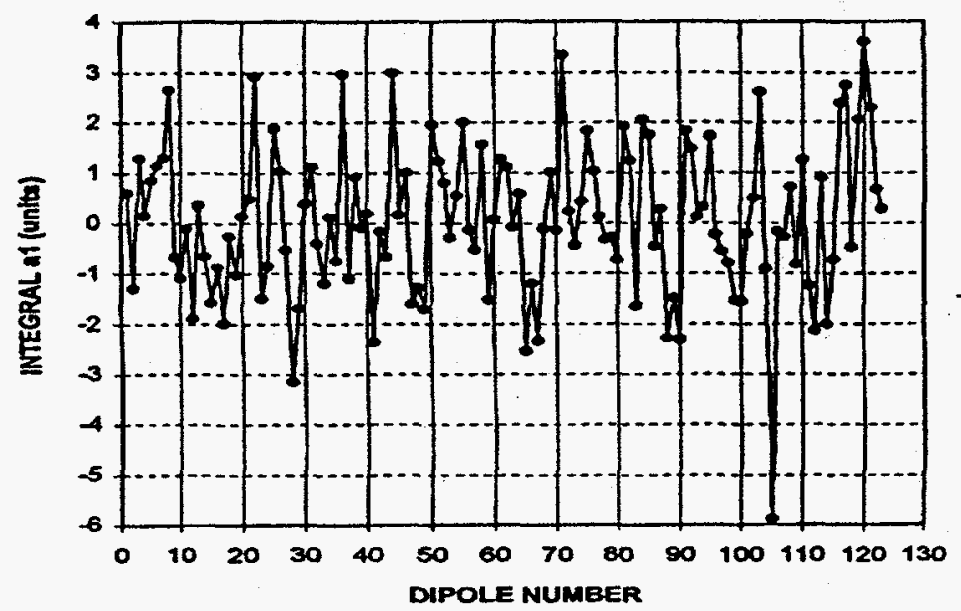

Fig. 7. Trend plot of arc dipole integral skew quadrupole

the change in coil cross section.)

The correlation of the warm and $5 \mathrm{kA}$ values of the skew

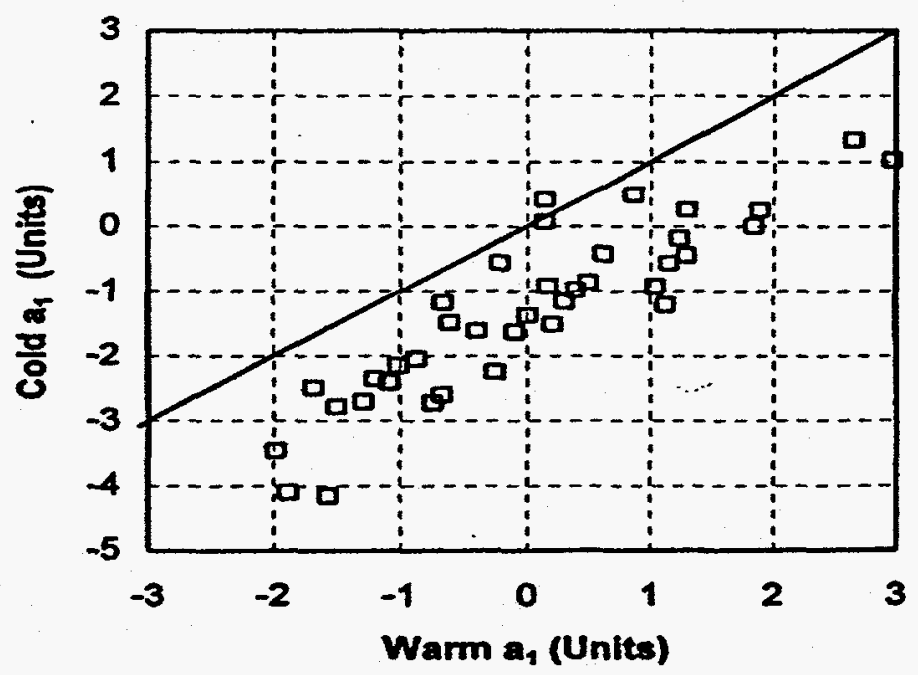

Fig. 8. Correlation of warm and $5 \mathrm{kA}$ measurements of arc dipole integral skew quadrupole 
quadrupole is shown in Figure 8. The rms variation of the warm cold difference is 0.6 units. Magnets with identical warm and cold measurements would lie on the line. At $5 \mathrm{kA}, \mathrm{a}_{1}$ is 1.6 units lower than at room temperature due to field leakage from the yoke and the asymmetric design of the position of the cold mass in the iron vacuum vessel. Efforts have been made during the production run to reduce $a_{1}$ at $5 \mathrm{kA}$ by introducing a compensating asymmetry in the weight of the yoke blocks [13]. Small changes in the allowed harmonics, $b_{2}, b_{4}$, etc. have been made during the production run. These are discussed in detail in another paper submitted to this Conference [14].

\section{ARC QUADRUPOLES}

\section{A. Design and Construction}

A cross section of the quadrupole is shown in Figure 9. The arcs will contain 276 quadrupoles of $80 \mathrm{~mm}$ aperture, $1.11 \mathrm{~m}$ magnetic length, and a gradient of $75 \mathrm{~T} / \mathrm{m}$ measured at the $5 \mathrm{kA}$ operating current. The insertion regions will contain 144 additional $80 \mathrm{~mm}$ quadrupoles with lengths ranging from $0.93 \mathrm{~m}$ to $1.81 \mathrm{~m}$. Including spares, NGC will produce $43280 \mathrm{~mm}$ quadrupole cold masses. Production is scheduled to be completed at the end of 1995. (Incorporation of the quadrupoles into a CQS assembly is done at BNL [3].)

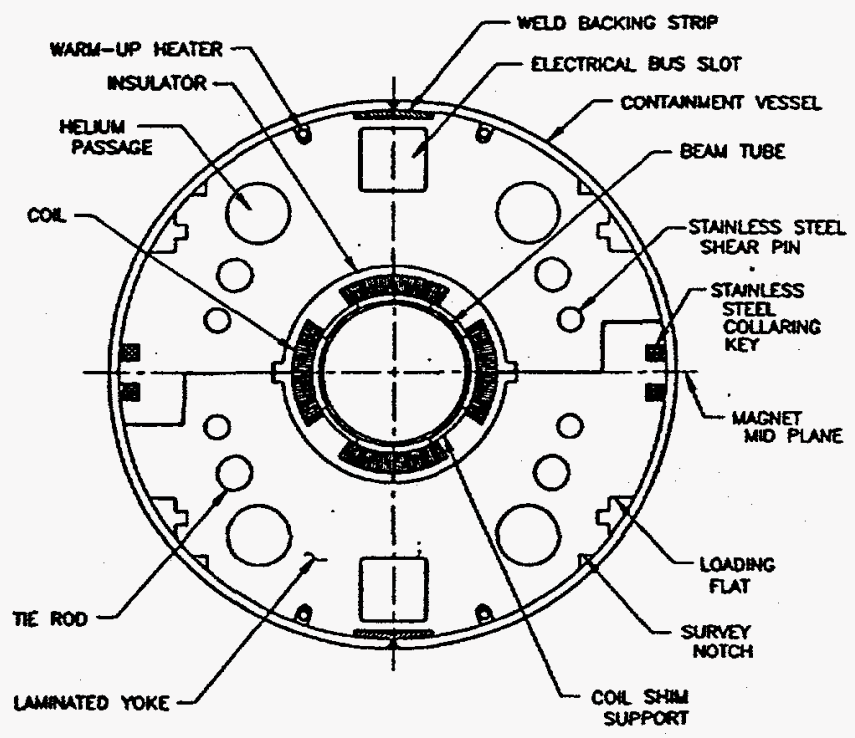

Fig. 9. Cross section of RHIC arc quadrupole cold mass

For reasons of economy, these magnets use many features of the dipole design, including the 30-strand cable with all-Kapton insulation, an injection-molded phenolic spacer between the coil and yoke, and a two-piece yoke with a tapered midplane and horizontal split. The yoke laminations are the same thickness as those used in the dipole. The single-layer coil has 16 turns and a symmetric copper wedge. Additional details of the magnet design are given elsewhere [15].

\section{B. Test Procedures and Results}

Cryogenic tests of the quadrupole cold masses were carried out in vertical dewars filled with liquid helium at $4.35 \mathrm{~K}$ and $1.12 \mathrm{~atm}$. The magnets were ramped at $83 \mathrm{~A} / \mathrm{sec}$ until they quenched. Magnets were quenched typically six times, to establish whether they were at the conductor limit. The quench performance was very good. All have quenched above $8 \mathrm{kA}$, with $90 \%$ reaching the conductor limit. All 58 tested so far have been accepted, with only one requiring individual review. Each of the initial 20 quads was cold-tested. Additional testing was done to confirm the effects of production changes. At present, $10 \%$ of the quads are being cold-tested.

The gradient and harmonics were measured with rotating coils long enough to obtain the integral with a single measurement, warm and cold. Warm measurements were carried out in a precision fixture built so that the axis of the quadrupole yoke and that of the measuring coil coincided within about $0.1 \mathrm{~mm}$. It is expected that a well-built magnet, mounted in this fixture, will have a vertical field angle, with a small offset due to the leads. For these quadrupoles, the average field angle was $-1.8 \mathrm{mrad}$, with an ms variation of $0.4 \mathrm{mrad}$. Similarly, it is expected that the axes of the field and the yoke will coincide. In the precise fixture the average horizontal offset was $0.03 \mathrm{~mm}$, with an rms variation of $0.06 \mathrm{~mm}$. The average vertical offset was $0.14 \mathrm{~mm}$, also with 0.06 $\mathrm{mm}$ ms variation. The vertical offset could be due to the magnet leads. These measurements indicate that the quadrupoles are precisely made. (The measurements used for installation are made after construction of the CQS as a whole.)

The mean value of the quadrupole integral transfer function measured warm is $16.5 \mathrm{~T} / \mathrm{kA}$, with an rms variation of $0.05 \%$, equal to the RHIC tolerance. The correlation between warm (10A) and cold values of the integral gradient is shown in Figure 10 . The cold values are higher than the warm by $0.07 \%$ and the fractional $\mathrm{rms}$ variation of the cold - warm difference is $0.035 \%$.

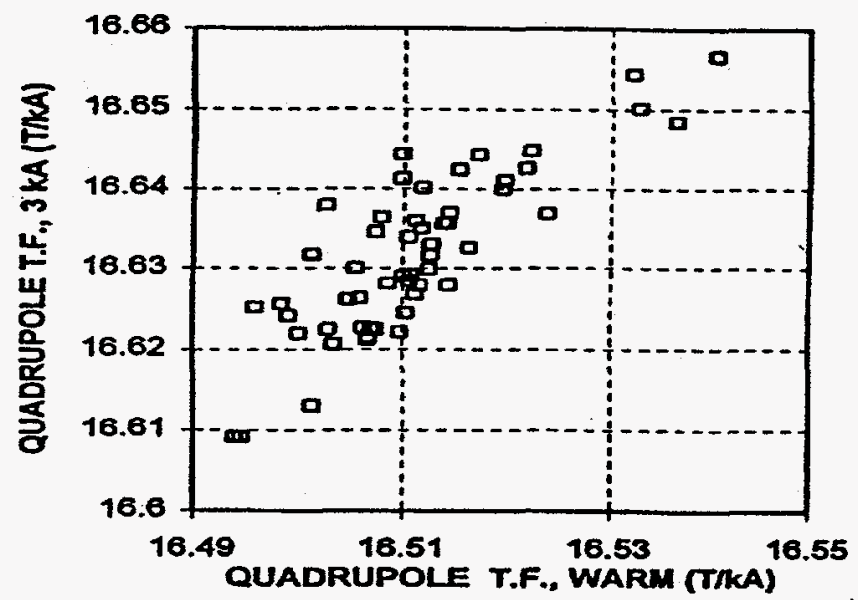

Fig. 10. Correlation of warm and $3 \mathrm{kA}$ measurements of arc quadrupole integral gradient

The average value of the first allowed harmonic in quadrupoles, the dodecapole $b_{5}$ is 1.4 units (warm). Shifts at the level of 0.5 units have occurred during the production run. A finite 
value is also expected for the octupole $b_{3}$ due to the dipole symmetry of the yoke. The average value of $b_{3}$ is -0.6 units (cold). However, five quadrupoles have $b_{3}$ in the range of 5 to 6 units due to misplacement of shims early in the production run. These magnets have been accommodated at special places in the lattice [12].

\section{ACKNOWLEDGEMENTS}

We acknowledge with pleasure the hard work and technical expertise of the Northrop Grumman staff.

\section{REFERENCES}

[1] P. Thompson et al., "Superconducting Sextupoles and Tuning Quadrupoles for RHIC, " Proc. 1995 International Particle Accelerator Conference (PAC'95), Dallas.

[2] A. Morgillo et al., "Superconducting $8 \mathrm{~cm}$ Corrector Magnets for the Relativistic Heavy Ion Collider (RHIC), "ibid.

[3] S. Mulhall et al., "Combined Element Magnet Production for the Relativistic Heavy Ion Collider (RHIC) at BNL," ibid.

[4] A. F. Greene et al., "Manufacture and Testing of the Superconducting Wire and Cable for the RHIC Dipoles and Quadrupoles," Proc. 1994 Applied Superconductivity Conf., Boston (to be published) and BNL Report No. BNL 60350. Corporation.

[5] Kapton is a registered trademark of the DuPont

[6] M. Anerella et al., "Improved Cable Insulation for Superconducting Magnets," Proc. 1993 Particle Accelerator Conference (Washington, D.C.), pp. 2790 - 2792.

Corporation.

[7] $\mathrm{RX}$ is a registered trademark of the Rogers

[8] Ultem is a registered trademark of the General Electric Corporation.

[9]P. Wanderer et al., "Dipole Magnet Development for the RHIC Accelerator," pp. 175 -187, New Techniques for Future Accelerators III, ed. G. Torelli, Plenum Press, New York, 1990.

[10] M. Anerella et al., "Industrial Production of RHIC Magnets," to be submitted to 1995 Magnet Technology Conference (MT14), Tampere, Finland.

[11] R. Thomas et al., "Performance of Field Measuring Probes for SSC Magnets," pp. 715-718, Supercollider 5, ed. P. Hale, Plenum Press, New York, 1994.

[12] J. Wei et al ., "Field Quality Evaluation of the Superconducting Magnets for the Relativistic Heavy Ion Collider," Proc. PAC'95.

[13] R. Gupta et al., "Skew Quadrupole in RHIC Dipole Magnets at High Fields," to be submitted to MT14, Tampere, Finland.

[14] R. Gupta et al., "Field Quality Control Through the Production Phase of the RHIC Arc Dipoles," Proc. PAC"95.

[15] P. Wanderer et al., "Test of Eight Superconducting Arc Quadrupoles for RHIC," IEEE Trans. Magnetics, Vol. 30, No. 4 (July 1994), p. 1734; P. Thompson et al ., "B Series RHIC Arc Quadrupoles," Proc. 1993 Particle Accelerator Conference, p.2766.

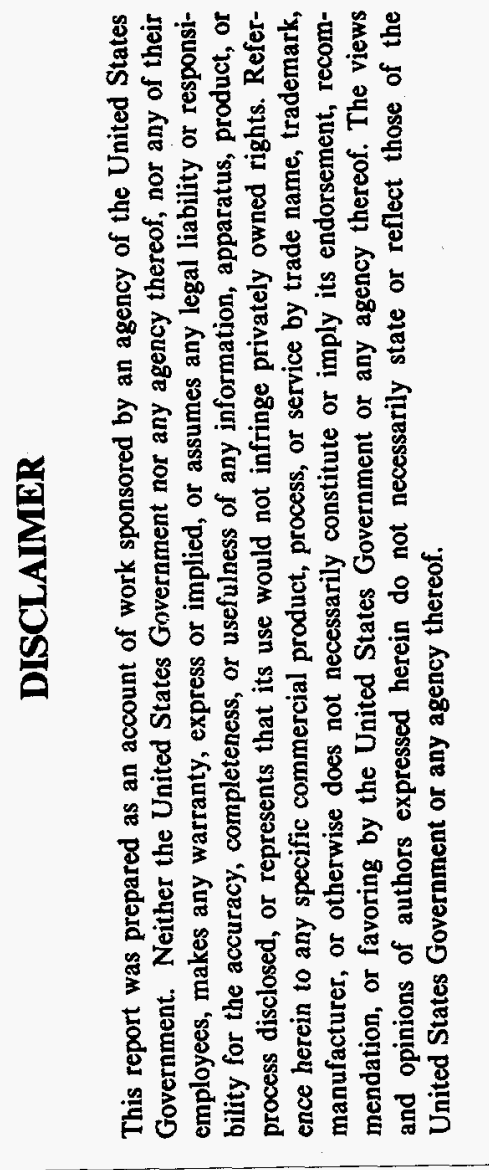

